\title{
Radial Extracorporeal Shock Wave Reduce Myogenic Contracture and Muscle Atrophy via inhibiting NF-kB/HIF-1a Signaling Pathway in Rabbit
}

Feng Wang ( $\square$ amor1993630@icloud.com )

The second hospital of Anhui Medical University https://orcid.org/0000-0003-4548-2309

\section{Wen Li}

The Second Affiliated Hospital of Wannan Medical college

\section{Yun Zhou}

The Second Hospital of Anhui Medical University

\section{Peng Peng Huang}

The Second Hospital of Anhui Medical University

\section{Research}

Keywords: Radial extracorporeal shock wave, muscle atrophy, myogenic contracture, NF-KB/HIF-1a signaling pathway, MyoD

Posted Date: January 25th, 2021

DOI: https://doi.org/10.21203/rs.3.rs-153164/v1

License: (c) (i) This work is licensed under a Creative Commons Attribution 4.0 International License. Read Full License

Version of Record: A version of this preprint was published at Connective Tissue Research on May 20th, 2021. See the published version at https://doi.org/10.1080/03008207.2021.1920934. 


\section{Abstract}

\section{Background}

Recent studies have shown that radial extracorporeal shock wave therapy (rESWT) modulates viability and gene expression of human skeletal muscle cells in vitro. However, the underlying biological effects and mechanisms of rESWT on muscle atrophy and myogenic contracture in animal mode have not yet been investigated.

\section{Objective}

To investigate the underlying biological effects and mechanisms of rESWT on myogenic contracture and muscle atrophy in a rabbit model of extending knee joint contracture.

\section{Methods}

Forty rabbits were randomly divided into eight groups. In group Control, the left knee joint was not fixed. In group I-4w, the left knee joint was only fixed for four weeks. In groups SR-1w, SR-2w, and SR-4w, the left knee joint was fixed for four weeks before the rabbits underwent one, two, and four weeks of self-recovery, respectively. In groups rESWT-1w, rESWT-2w, and rESWT-4w, the left knee joint was fixed for four weeks before the rabbits underwent one, two, and four weeks of rESWT, respectively. The degree of myogenic contracture was measured, the cross-sectional area and key protein levels for NF-KB/HIF-1a signaling pathway and myogenic regulatory factors of the rectus femoris were evaluated.

\section{Results}

During the recovery period, biological findings in this study showed that the levels of myogenic contracture and muscle atrophy were milder in group rESWT by compared with group SR at the same time point. Molecular biological analysis in the present study showed that MyoD protein levels in the group rESWT was significantly higher than those in the group SR, and importantly, phospho-NF-kB p65 and HIF-1a protein levels in the group rESWT were significantly lower than those in the group SR at the same time point.

\section{Conclusions}

Our study demonstrates that rESWT has the potential to reduce myogenic contracture and muscle atrophy after long-term immobilization in animal model. It is a possible mechanism that changing the low oxygen environment in skeletal muscle through rESWT may inhibit activation of NF-KB/HIF-1a signaling pathway, thereby enhancing the expression of myogenic regulatory factors.

\section{Background}


Long-term immobilization is a major cause of joint contracture in humans globally, and reversing established joint contracture following fixing is of great clinical challenge [1]. The two structural components contributing to joint contracture formation are myogenic and arthrogenic contracture [2]. Skeletal muscle atrophy is an important part of myogenic contracture [3]. Long-term immobilization also leads to skeletal muscle atrophy, characterized by loss of tissue protein because of decreased synthesis and increased degradation, an increase in the amount of intramuscular connective tissue, and the arrangement of collagen fibrils in the endomysium [4]. Because of the importance of skeletal muscle atrophy in myogenic contracture pathology, understanding of this process is helpful to identify therapeutic targets for myogenic contracture.

More than $90 \%$ of the energy produced by muscle cells comes from the aerobic pathway. A reduction in $\mathrm{O}_{2}$ availability would therefore challenge skeletal muscle homeostasis [5]. Previous study revealed that overexpression of hypoxia-inducible factor (HIF)-1a and skeletal muscle hypoxia are the biological processes underlying skeletal muscle atrophy induced by immobilization [6]. HIF-1a is the master transcription factor in response to cell hypoxia. In response to hypoxia, activated HIF-1a translocates to the nucleus and initiates target gene transcription [7]. The nuclear factor-kappa B (NF-KB) family is a family of transcription factors that act as crucial regulators in inflammatory diseases. Moreover, crosstalk between HIF-1 $\mathrm{a}$ and NF-KB controls the response in a variety of medical conditions [8]. Skeletal muscle tissue possesses a remarkable capacity to regenerate in response to muscle injury. Adult myofibers are terminally differentiated so that muscle repair is largely attributed to satellite cells [9]. From previous reports, silencing S100B expression in myoblast cell lines by RNA interference resulted in reduced NF-KB activity and enhanced MyoD, myogenin and MyHC expression and myotube formation [10]. Therefore, therapeutic approaches for treating skeletal muscle atrophy should thus address ways to inhibit the overexpression of HIF-1a and hypoxia in the immobilized skeletal muscle.

Radial extracorporeal shock wave therapy (rESWT) is served as a non-invasive therapeutic option for skeletal muscle disease in recent years. It is generally known that rESWT can improve microcirculation, inhibit local inflammation, stimulate cell proliferation and regeneration, and promote recruitment of endothelial progenitor cells and mesenchymal stem cells at the injured tissue [11]. Previously, it has been reported that rESWT stimulates and accelerates regenerative processes of acute muscle injuries in adult Sprague-Dawley rats [12]. From previous reports, exposure to radial extracorporeal shock waves modulates viability and gene expression of human skeletal muscle cells. The results demonstrate a dosedependent effect of rESWT on gene expression of muscle-specific genes contained Pax7, NCAM, Myf5, and MyoD [13]. It is worth mentioning that MyoD is one of the earliest markers of myogenesis [14]. MyoD is a key component of ubiquitin-proteasome-dependent proteolysis pathway, as it is one of the myogenic regulatory factors that plays a crucial role in inhibiting muscle proteolysis [15].

Although there are some reports that indicate the therapeutic effect of skeletal muscle atrophy in vitro, the biological mechanisms in animal of rESWT causing these noticed effects have remained largely unknown. Our early work has allowed us to develop a rabbit model of extending knee joint contracture in which we have successfully described changes in the structure of shrinking muscle [16]. Importantly, our 
previous study that evaluated the effect of radial extracorporeal shock wave combined with ultrashort wave diathermy on fibrosis and contracture of muscle found that rESWT reduces myofibrosis by inhibited the expression of HIF-1 $\alpha$ and transforming growth factor (TGF)- $\beta 1$ in the rectus femoris [11]. Later, we speculate that rESWT would affect the NF-KB/HIF-1a signaling pathway in skeletal muscle (Figure 1). Furthermore, our subsequent muscle tissue analysis found that rESWT effectively reduces muscle atrophy, and so we performed the present study. The aim of the present study was to preliminarily investigate biological effects of rESWT on myogenic contracture and muscle atrophy in a rabbit model of extending knee joint contracture, and to investigate the possible mechanism of action.

\section{Methods}

\section{Animals}

Animal care and experimental procedures were performed in accordance with the Guidelines for Animal Experimentation of Anhui Medical University and were approved by the Institutional Animal Care and Use Committee (LLSC20190761). Forty 4-month-old male, skeletally mature New Zealand white rabbits were obtained from Anhui Medical University Experimental Animal Center (Hefei, China). The rabbits were randomly divided into the following eight groups: Control, l-4w, SR-1 w, SR-2w, SR-4w, rESWT-1w, rESWT-

$2 \mathrm{w}$, and rESWT- $4 \mathrm{w}$, and housed one per cage at $22^{\circ} \mathrm{C}-24^{\circ} \mathrm{C}$ with a $12-\mathrm{hr}$ light/dark cycle and were allowed access to food and water ad libitum. All rabbits were anesthetized by intravenous administration of 30 $\mathrm{mg} / \mathrm{kg}$ sodium pentobarbital in an ear vein.

\section{Intervention methods}

In group Control, the left knee joint was not fixed to serve as a control. In group I-4w, the left knee joint was only fixed for four weeks at full extension using a plaster cast from groin to proximal toes as described previously (Figure 2. A) [16], and did not undergo recovery. In groups SR-1w, SR-2w, and SR-4w, the left knee joint was fixed for four weeks before the rabbits underwent one, two, and four weeks of selfrecovery, respectively, without any special rehabilitation protocol. In groups rESWT-1w, rESWT-2w, and rESWT-4w, the left knee joint was fixed for four weeks before the rabbits underwent one, two, and four weeks of rESWT, respectively. As in our previous study, A radial shock wave device (DolorClast, Switzerland) was used in the experiment, once in a week (Figure 2. C) [11]. Firstly, each rabbit received less than 500 times impulses of shock waves at a small dose of $1.5 \mathrm{bar}, 4 \mathrm{~Hz}$ to the left quadriceps femoris area, in order to adapt the rabbits to the stimulation. Secondarily, the rabbit received 2000 times impulses at an experimental dose of $2.5 \mathrm{bar}, 6 \mathrm{~Hz}$ to the same area.

\section{Measurement of myogenic contracture}

The rabbits were euthanized with an excess amount of sodium pentobarbital. The left hindlimb was dislocated at the left hip joint and completely removed. As in our previous study, the range of motion (ROM) of the left knee joint before and after myotomy was measured, respectively [16,17]. In accordance 
with the method described by Trudel and Uhthoff [18], we evaluated the myogenic contracture caused by the muscular structures. The following formulas were used to calculate the degree of contracture.

Decrement in ROM as a result of total contracture = ROM before myotomy (of the control knee) - ROM before myotomy (of the contracted knee)

Decrement in ROM as a result of arthrogenic contracture $=$ ROM after myotomy (of the control knee) ROM after myotomy (of the contracted knee)

Decrement in ROM as a result of myogenic contracture $=$ Decrement in ROM as a result of total contracture - Decrement in ROM as a result of arthrogenic contracture

\section{Measurement of muscle fiber cross-sectional area}

After measuring ROM, two muscle tissue samples $(1 \mathrm{~cm} \times 1 \mathrm{~cm} \times 0.5 \mathrm{~cm}$ in size) were cut from the middle of the rectus femoris. One sample was stained with hematoxylin and eosin, while the other was frozen in liquid nitrogen at $-80^{\circ} \mathrm{C}$ until histological analysis.

In the rectus femoris sample stained with hematoxylin and eosin, the cross-sectional area (CSA) of individual myofibers was photographed using a TE2000-U inverted microscope (Nikon Corporation, Tokyo, Japan) and measured using Image $J$ software (National Institutes of Health, USA). Six randomly selected fields of view were analyzed in each group.

\section{Western Blot analysis}

The skeletal muscle samples were ground into powder with liquid nitrogen using a grinder and homogenized in RIPA buffer (Beyotime, China) containing protease inhibitors at four degrees Celsius. Homogenates were centrifuged at $12,000 \times \mathrm{g}$ for 30 min three times at four degrees Celsius, and the resulting supernatants were collected. The protein concentrations were determined using the bicinchoninic acid method. Protein lysates were separated on a $10 \%$ sodium dodecyl sulfatepolyacrylamide electrophoresis gel and transferred on to polyvinylidene fluoride membranes (Millipore, USA). After being blocked with five-percent non-fat dry milk in Tris-buffered saline Tween-20 (TBST) at room temperature for two hours, the membranes were incubated with rabbit anti-phospho-NF-kB p65 antibody (dilution 1:1000; Cell Signaling, USA), rabbit anti-HIF-1a antibody (dilution 1:1000; Cell Signaling, USA) and rabbit anti-MyoD mAb (dilution 1:1000; Cell Signaling, USA) at four degrees Celsius overnight. On the second day, after being washed in TBST solution three times for $10 \mathrm{~min}$ per wash, the membranes were incubated with peroxidase-conjugated affinipure goat anti- rabbit IgG-HRP (dilution 1:3000; Cell Signaling, USA) as the secondary antibody for two hours at room temperature. After being washed three times with TBST for 10 min per wash, the membranes were evaluated with the enhanced chemiluminescence system in accordance with the manufacturer's instructions. The band densities were quantified using Image $\mathrm{J}$ software (National Institutes of Health, USA). The relative protein levels were calculated by comparison with the amount of GAPDH (dilution 1:1000; Cell Signaling, USA) as a loading control. 


\section{Statistical analysis}

All data are expressed as the mean \pm standard error of the mean. The assumptions of normality of data and homogeneity of variances between the groups were analyzed by SPSS 21.0 (Chicago, IL, USA). Differences in the myogenic contracture, CSA, average protein levels for HIF-1a and MyoD between group SR and group rESWT at each recovery timepoint were assessed using the Student's t-test. A significant difference between group SR and group rESWT at the same timepoint was considered to be present when the $95 \%$ confidence interval did not overlap with zero. One-way analysis of variance (ANOVA) and the Tukey-Kramer test were performed to examine differences between the timepoints in the myogenic contracture, CSA, average protein levels for phospho-NF-kB p65, HIF-a and MyoD.

\section{Results}

During the immobilization period, one rabbit in group I-4w died from anesthetic stress. All other rabbits survived the recovery period. No rabbit developed prolonged edema or acute inflammation.

\section{Myogenic contracture}

The myogenic contracture was significantly greater in group I-4w compared with group Control $(P<0.05$, Figure 3). The difference in myogenic contracture between group Control $\left(0 \pm 0^{\circ}\right)$ and group $1-4 \mathrm{w}(36.2 \pm$ $0.9^{\circ}$ ) was $36.2 \pm 0.9^{\circ}$. After four weeks of immobilization, serious myogenic contracture developed. Oneway ANOVA and the Tukey-Kramer test applied to the resulting curves indicated that the myogenic contracture of group rESWT and group SR decreased in tandem with the prolongation of recovery time ( $P$ $<0.05$, Figure 3 ). After four weeks of recovery, the myogenic contracture in both group rESWT and group $\mathrm{SR}$ were restored to varying degrees. The myogenic contracture was significantly reduced in group rESWT compared with group SR at the same timepoint $(P<0.05$, Figure 3$)$. Therefore, the rabbits that received rESWT tended to have better recovery of the myogenic contracture than those receiving only natural recovery.

\section{Muscle fiber cross-sectional area}

The CSA values were significantly reduced in group I-4w compared with group Control $(P<0.05$, Figure 4$)$. After four weeks of immobilization, serious skeletal muscle atrophy formed. The difference in CSA values between group Control $\left(5230.8 \pm 16.07 \mu \mathrm{m}^{2}\right)$ and group l-4w $\left(4395.0 \pm 22.9 \mu \mathrm{m}^{2}\right)$ was $835.8 \pm 27.98 \mu \mathrm{m}^{2}$. One-way ANOVA and the Tukey-Kramer test applied to the resulting curves indicated that the CSA values in group rESWT and group SR increased in tandem with the prolongation of recovery time $(P<0.05$, Figure 4). After four weeks of recovery, the skeletal muscle atrophy in both group rESWT and group SR were restored to varying degrees. The CSA values were significantly increased in group rESWT compared with group SR at the same timepoint $(P<0.05$, Figure 4$)$. Therefore, the rabbits that received rESWT tended to have better recovery of skeletal muscle atrophy than those that received only natural recovery.

\section{Changes in NF-kB/HIF-1a signaling pathwayexpression}


The average protein levels for phospho-NF-KB p65 and HIF-a were significantly greater in group I-4w compared with group Control $(P<0.05$, Figure 5). Oneway ANOVA and the Tukey-Kramer test applied to the resulting curves indicated that the average protein levels for phospho-NF-KB p65 and HIF- $\alpha$ in group rESWT decreased with the prolongation of recovery time. It also suggested that the average protein levels for phospho-NF-KB p65 and HIF-a in group SR decreased from the second week $(P<0.05$, Figure 6$)$. The average protein levels for phospho-NF-KB p65 and HIF-a were significantly reduced in group rESWT compared with group SR at the same timepoint $(P<0.05$, Figure 5$)$. Therefore, the rabbits that received rESWT tend to cause more decrease in the expression of phospho-NF-KB p65 and HIF-a than those that received only natural recovery.

\section{Changes in the expression of MyoD}

The average protein levels for MyoD were significantly greater in group I-4w compared with group Control $(P<0.05$, Figure 6). Oneway ANOVA and the Tukey-Kramer test applied to the resulting curves indicated that the average protein levels for MyoD in group rESWT and group SR increased in tandem with the prolongation of recovery time, except in the first week $(P<0.05$, Figure 6$)$. After four weeks of recovery, the MyoD protein expression in both group rESWT and group SR had increased to varying degrees. The average protein levels for MyoD were significantly reduced in group rESWT compared with group SR at the same timepoint $(P<0.05$, Figure 6$)$. Therefore, the rabbits that received rESWT tend to cause more increase in the expression of MyoD than those that received only natural recovery.

\section{Discussion}

Myogenic contracture is a common reason for joint dysfunction. It is well known that reducing the degree of muscle atrophy and myofibrosis is an effective treatment measure for myogenic contracture, but the treatment modalities are still limited. Extracorporeal shock wave has been applied for the treatment of non-invasive procedure in various musculoskeletal disorders over the last two decades [19]. Although there are some reports that indicate the osteogenic effect of rESWT [20, 21], but the effect and biological mechanisms of rESWT for myogenic contracture and muscle atrophy have remained largely unknown. Previous research has been isolated and cultured primary human skeletal muscle cells from biopsy specimens and investigated the effect of rESWT on human skeletal muscle cells [13]. It is also the first research to provide evidence that rESWT has the potential to modulate the biological function of primary human muscle cells. In order to further investigate the effect of rESWT on muscle atrophy and myogenic contracture, we designed this experiment.

Biological findings in this study showed greater levels of myogenic contracture of group l-4w than that in control. It is suggesting that there was serious myogenic contracture after 4 weeks of immobilization. In general, both group rESWT and group SR were obtained recovery on different levels. In addition, in each group rESWT at the same time, the levels of myogenic contracture were milder than that observed in the group SR. Relatively, our results of histological findings supported the biological findings. Thus, it seemed that some certain effects by rESWT significantly prevented the decrease in muscle fiber cross-sectional 
area that is typically induced by immobilization. Previous research found that mechanical stimulation of shock wave can improve the oxygen saturation of local tissue and promote damaged tissue regeneration [22]. Moreover, our previous study also showed that the protein levels of HIF-1a was significantly lower in rabbits rectus femoris after 4 weeks of rESWT than that in group non-treatment [11].

Although NF-KB are key transcription factors involved in inflammation diseases, hypoxia also causes the activation of NF-KB through the phosphorylation of IKB a on tyrosine residues [23]. NF-KB is a direct modulator of HIF-1a expression. The HIF-1a promoter is responsive to selective NF-KB subunits [24]. Moreover, previous research also found that hypoxia up-regulates HIF-1a transcription by involving PI3K and NF-KB in pulmonary artery smooth muscle cells [25]. Consistent with these previous studies, the protein levels for phospho-NF-KB p65 and HIF-a of the rectus femoris in the immobilized knee obviously increased during 4 weeks of immobilization when compared with group Control. Thus, it was preliminarily suggested that there is activation of NF-KB/HIF-1a signaling pathway. Apart from this, during the recovery period, the protein levels for phospho-NF-KB p65 and HIF-a of the rectus femoris exhibited different tendencies in group rESWT compared with group SR. Importantly, the molecular biological analysis in the present study showed that phospho-NF-KB p65 and HIF-1 a protein levels in the group rESWT were significantly lower than those in the group SR at the same time point, so we considered that NF-KB/HIF-1a signaling pathway can be inhibited by rESWT through changing the low oxygen environment in rectus femoris.

Like our previous studies, the protein levels for MyoD increased during 4 weeks of immobilization when compared with group Control, and the average protein levels for MyoD in group SR-1w and group rESWT$1 \mathrm{w}$ both showed declining and stable trends compared with group I-4w [16]. The former was suggested that MyoD protein expression was compensatoryly elevated to counteract the effect of muscle atrophy during the immobilization process, but this compensation was not enough to offset muscle atrophy. The latter was illustrated that muscle atrophy worsens during recovery in correlation with proteolysis, and apoptosis. In agreeance with previous research, we also found that there is a significant increasing of the MyoD protein expression when the HIF-1a protein expression was decreased in group rESWT, which was consistent with the effects on myogenic contracture and muscle fiber cross-sectional area, indicating that rESWT may partly influence the expression of MyoD and thus ameliorate myogenic contracture and muscle atrophy. The expression of HIF-1a synchronized with that of myogenic regulatory genes during muscle regeneration at both the mRNA and protein levels. Previous research suggested that the HIF-1a protein was localized in the nucleus and cytoplasm of the majority of myoblasts and myotubes in myogenic cell culture, and the MyoD proteins expression increased concomitant with the HIF-1a protein decreased expression of after the induction of myogenic differentiation [26].

These recovery effects on immobilized-induce myogenic contracture and muscle atrophy of group SR in this study were also similar to our previous study [16]. Although there was non treatment, we also found that there was a certain degree of recovery of myogenic contracture and muscle atrophy in group SR. MyoD is a key component of the proteolytic pathway, as it is one of the myogenic regulatory factors that 
plays a crucial role in inhibiting muscle proteolysis, but it is not only regulated by NF-KB/HIF-1a signaling pathway.

This study has certain limitations. First, our results are limited to an animal model and cannot be generalized to humans. Next, due to experimental conditions, our data for the expression of genes related to NF-KB/HIF-1a signaling pathway are limited. rESWT induced specific NF-KB signaling pathway inhibition mode should be considered in future research. At last, due to the lack of uniform standards, the best dose of the rESWT was only based on clinical experiencenot and not discussed in detail. The most suitable dose also should be considered in future research.

\section{Conclusions}

Our study demonstrates that rESWT has the potential to reduce myogenic contracture and muscle atrophy after long-term immobilization in animal model. It is a possible mechanism that changing the low oxygen environment in skeletal muscle through rESWT may inhibit activation of NF-KB/HIF-1a signaling pathway, thereby enhancing the expression of myogenic regulatory factors. Based on our experimental findings, we hypothesize that rESWT could be a promising therapeutic modality to ameliorate myogenic contracture and muscle atrophy after long-term immobilization in human.

\section{Abbreviations}

HIF: hypoxia-inducible factor, NF-kB: nuclear factor-kappa B, rESWT: radial extracorporeal shock wave therapy, TGF: transforming growth factor, ROM: range of motion; CSA: cross-sectional area, MyoD: myogenic differentiation.

\section{Declarations}

\section{Ethics approval and consent to participate.}

Animal care and experimental procedures were performed in accordance with the Guidelines for Animal Experimentation of Anhui Medical University and were approved by the Institutional Animal Care and Use Committee (LLSC20190761).

\section{Consent for publication}

Not applicable.

\section{Availability of data and material}

The datasets used and analysed during the current study are available from the corresponding author on reasonable request. All data generated or analysed during this study are included in this published article. The manuscript, including related data, figures and tables have not been previously published and are not under consideration elsewhere. 


\section{Competing interests}

The authors declare that they have no competing interests.

\section{Funding}

This study was jointly funded projects of Wannan Medical College Research Foundation (WK2020ZF23) and Anhui Key Research and Development Program (201904a07020067).

\section{Authors' contributions}

Feng Wang conceived of the study and participated in its design and coordination and drafted the manuscript. Wen Li participated in the design of the study and performed the statistical analysis. Peng Peng Huang carried out the molecular studies. Yun Zhou participated in its design and coordination and helped to draft the manuscript. All authors read and approved the final manuscript.

\section{Acknowledgements}

We thank Professor Hua Wang from School of Public Health, Anhui Medical University for his valuable corrections and advices.

\section{References}

1. Wong K, Trudel G, Laneuville $\mathrm{O}$. Noninflammatory joint contractures arising from immobility: animal models to future treatments. BioMed Res Int. 2015; 2015(3): 848290.

2. Trudel G, Laneuville $\mathrm{O}$, Coletta E, et al. Quantitative and temporal differential recovery of articular and muscular limitations of knee joint contractures; results in a rat model. J Appl Physiol. 2014; 117: 730737.

3. Wang F, Zhang QB, Zhou Y, et al. The mechanisms and treatments of muscular pathological changes in immobilization-induced joint contracture: A literature review. Chin J Traumatol. 2019;22: 93-98.

4. Järvinen TA, Józsa $L$, Kannus $P$, et al. Organization and distribution of intramuscular connective tissue in normal and immobilized skeletal muscles. J Muscle Res Cell Motil. 2002; 23: 245-254.

5. Favier FB, Britto FA, Freyssenet DG, et al. HIF-1-driven skeletal muscle adaptations to chronic hypoxia: Molecular insights into muscle physiology. Cell Mol Life Sci. 2015; 72(24): 4681-4696.

6. Wagatsuma A. Effect of hindlimb unweighting on expression of hypoxia-inducible factor-1 alpha vascular endothelial growth factor, angiopoietin, and their receptors in mouse skeletal muscle. Physiological Research. 2008; 57(4): 613-620.

7. Peng Y, Fang Z, Liu M, et al. Testosterone induces renal tubular epithelial cell death through the HIF1a/BNIP3 pathway. J Trans Med. 2019; 17(1): 62.

8. Yu LM, Zhang WH, Han XX, et al. Hypoxia-induced ROS contribute to myoblast pyroptosis during obstructive sleep Apnea via the NF-KB/HIF-1a Signaling Pathway. Oxid Med Cell Longev. 2019; 2019: 
4596368.

9. Hawke TJ, Garry Myogenic satellite cells: physiology to molecular biology. J Appl Physiol. 2001; 91(2): 534-51.

10. Tubaro C, Arcuri C, Giambanco I, et al. S100B protein in myoblasts modulates myogenic differentiation via NF-kappaB-dependent inhibition of MyoD expression. J Cell Physiol. 2010; 223(1): 270-282.

11. Huang PP, Zhang QB, Zhou Y, et al. Effect of Radial Extracorporeal Shock Wave Combined with Ultrashort Wave Diathermy on Fibrosis and Contracture of Muscle. Am J Phys Med Rehabil. 2020; doi: 1097/PHM.0000000000001599.

12. Zissler A, Steinbacher P, Zimmermann R, et al. Extracorporeal shock wave therapy accelerates regeneration after acute skeletal muscle injury. Am J Sports Med 2017; 45: 676-84.

13. Mattyasovszky SG, Langendorf EK, Ritz U, et al. Exposure to radial extracorporeal shock waves modulates viability and gene expression of human skeletal muscle cells: a controlled in vitro study. $J$ Orthop Surg Res. 2018; 13(1): 75.

14. Bodine SC, Latres E, Baumhueter S, et al. Identification of ubiquitin ligases required for skeletal muscle atrophy. Science. 2001;294: 1704-1708.

15. Camerino GM, Desaphy JF, De Bellis M, et al. Effects of Nandrolone in the Counteraction of Skeletal Muscle Atrophy in a Mouse Model of Muscle Disuse: Molecular Biology and Functional Evaluation. PLoS ONE. 2015; 10: e0129686.

16. Wang F, Zhang QB, Zhou Y, et al. Effect of ultrashort wave treatment on joint dysfunction and muscle atrophy in a rabbit model of extending knee joint contracture: Enhanced expression of myogenic differentiation. Knee. 2020; 27(3): 795-802.

17. Zhang QB, Zhou Y, Zhong HZ, et al. Effect of stretching combined with ultrashort wave diathermy on joint function and its possible mechanism in a rabbit knee contracture model. Am J Phys Med Rehabil. 2018; 97: 357-363.

18. Trudel G, Uhthoff HK. Contractures secondary to immobility: is the restriction articular or muscular? An experimental longitudinal study in the rat knee. Arch Phys Med Rehabil. 2000; 81: 6-13.

19. Schmitz C, Csaszar NB, Milz S, et al. Efficacy and safety of extracorporeal shock wave therapy for orthopedic conditions: a systematic review on studies listed in the PEDro database. Br Med Bull. 2015; 116(1): 115-138.

20. Hofmann A, Ritz U, Hessmann MH, et al. Extracorporeal shock wave-mediated changes in proliferation, differentiation, and gene expression of human osteoblasts. J Trauma. 2008; 65 (6): 1402-1410.

21. Alshihri A, Niu W, Kämmerer PW, et al. The effects of shock wave stimulation of mesenchymal stem cells on proliferation, migration, and differentiation in an injectable gelatin matrix for osteogenic regeneration. J Tissue Eng Regen Med. 2020; 14(11): 1630-1640.

22. Kraemer R, Sorg H, Forstmeier V, et al. Immediate dose-response effect of high-energy versus lowenergy extracorporeal shock wave therapy on cutaneous microcirculation. Ultrasound Med Biol. 
2016; 42(12): 2975-2982.

23. Hayden MS, Ghosh S. Shared principles in NF-kappaB signaling. Cell 2008; 132(3): 344-362.

24. van Uden P, Kenneth NS, Rocha S. Regulation of hypoxia-inducible factor-1alpha by NF-kappaB. Biochem J. 2008; 412(3): 477-84.

25. Belaiba RS, Bonello S, Zähringer $C$, et al. Hypoxia up-regulates hypoxia-inducible factor-1alpha transcription by involving phosphatidylinositol 3-kinase and nuclear factor kappaB in pulmonary artery smooth muscle cells. Mol Biol Cell. 2007; 18(12): 4691-4697.

26. Vazeille E, Codran A, Claustre A, et al. The ubiquitin-proteasome and the mitochondria-associated apoptotic pathways are sequentially downregulated during recovery after immobilization-induced muscle atrophy. Am J Physiol Endocrinol Metab. 2008; 295: 1181-1190.

\section{Figures}

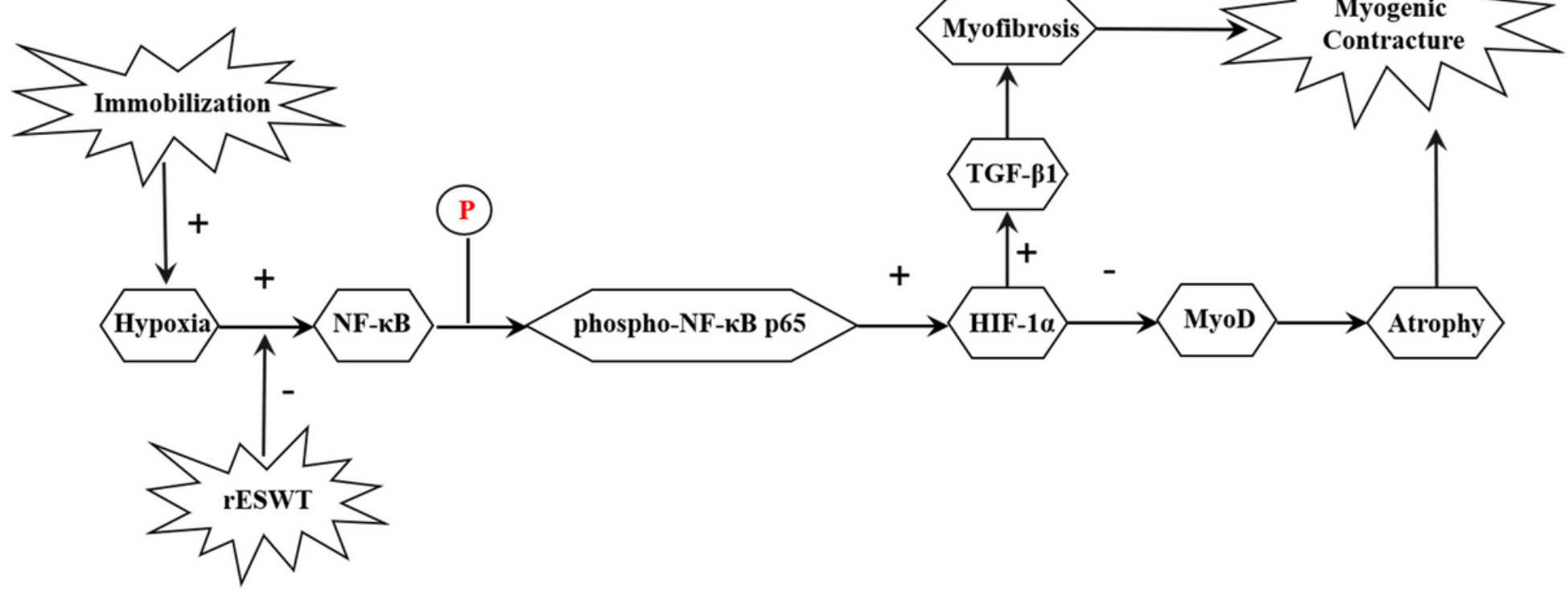

\section{Figure 1}

Research hypothesis.
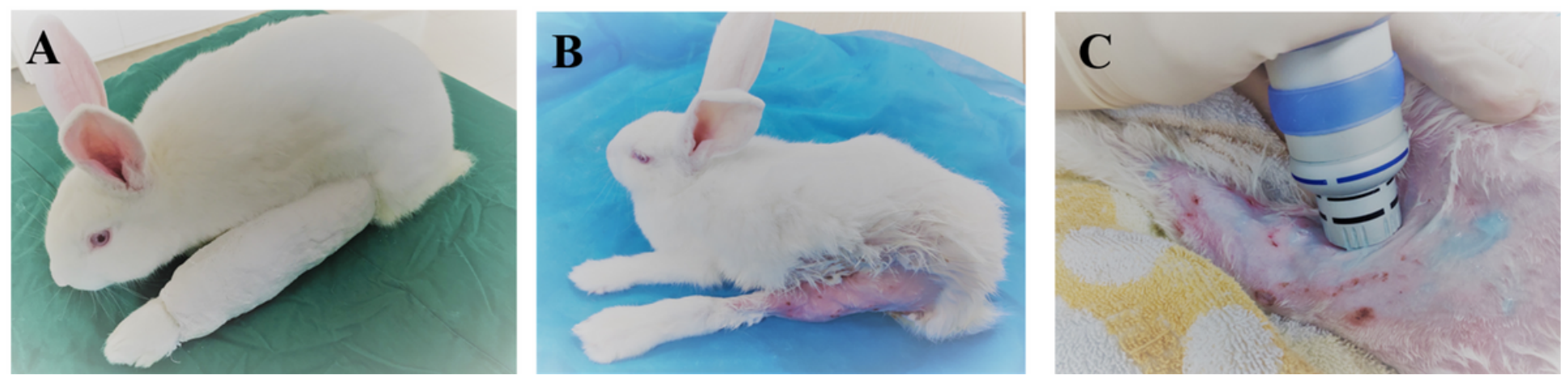
(A) Unilateral immobilization of the rabbit knee joint at full extension using a plaster cast from the groin to the proximal toes. (B) After four weeks of immobilization, serious myogenic contracture developed. (C) Radial extracorporeal shock wave therapy on quadriceps.

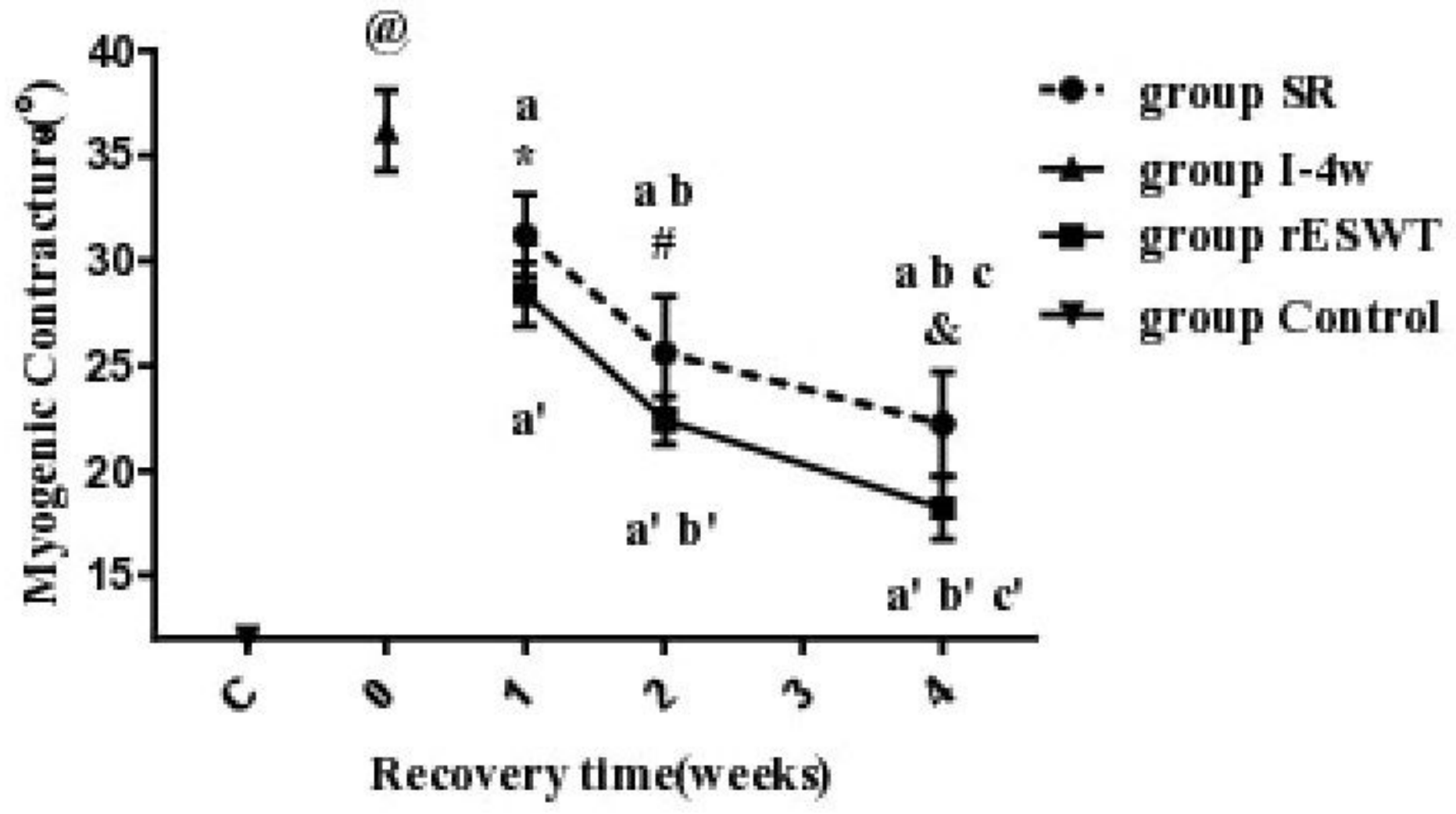

Figure 3

Effects of self-recovery and rESWT on the myogenic contracture. @P $<.05$ vs. group Control, aP $<.05$ vs. group $\mathrm{I}-4 \mathrm{w}, \mathrm{bP}<.05$ vs. group rESWT-1w, $\mathrm{CP}<.05$ vs. group rESWT-2w, a'P $<.05$ vs. group $\mathrm{I}-4 \mathrm{w}, \mathrm{b}^{\prime} \mathrm{P}<0.05$ vs. group SR-1W, $C^{\prime} P<0.05$ vs. group $S R-2 w ;{ }^{*} P<.05$ vs. group $S R-1 w$, \#P<.05 vs. group $S R-2 W, \& P<.05$ vs. group SR-4W. 
A
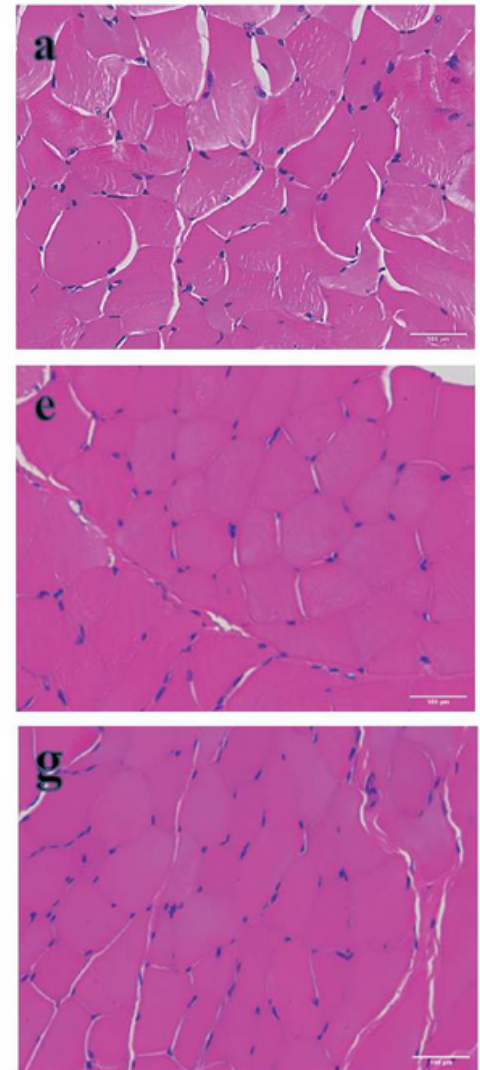
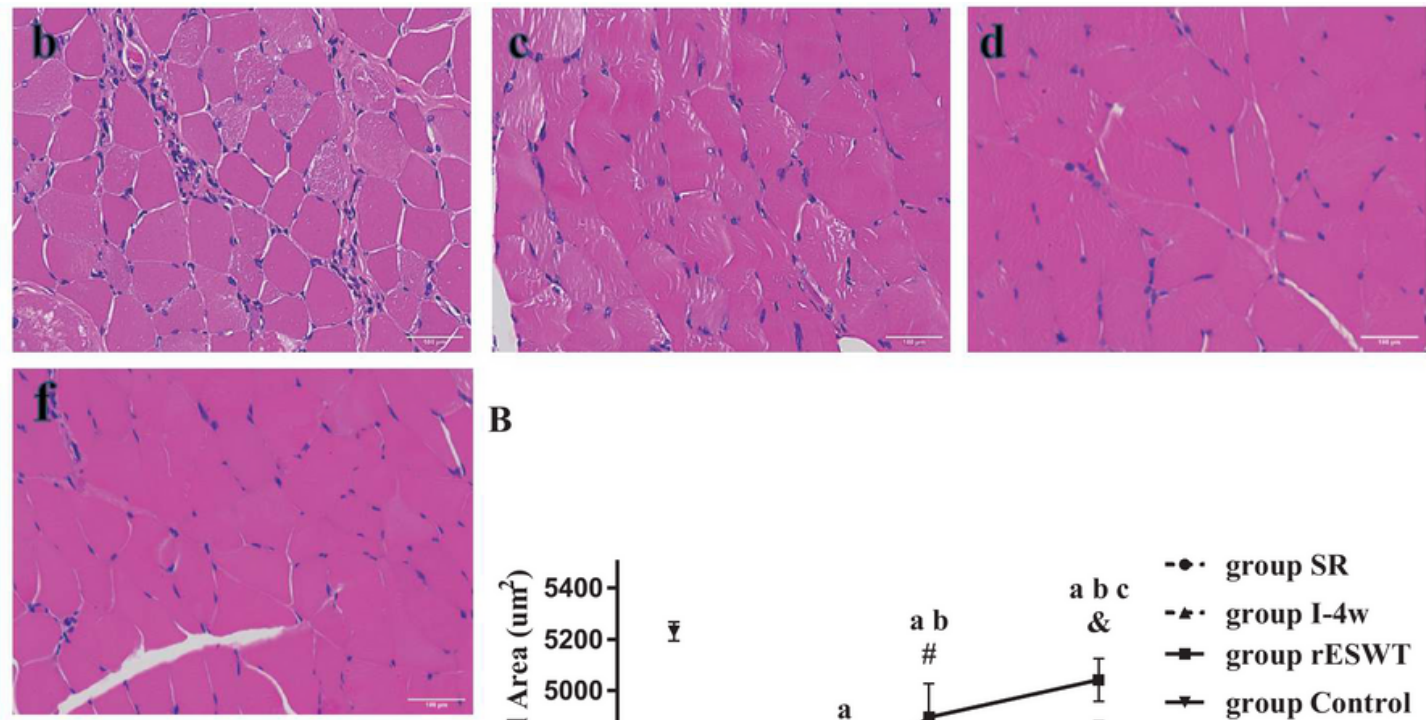

h

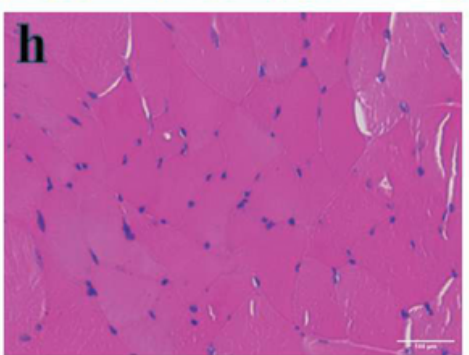

B

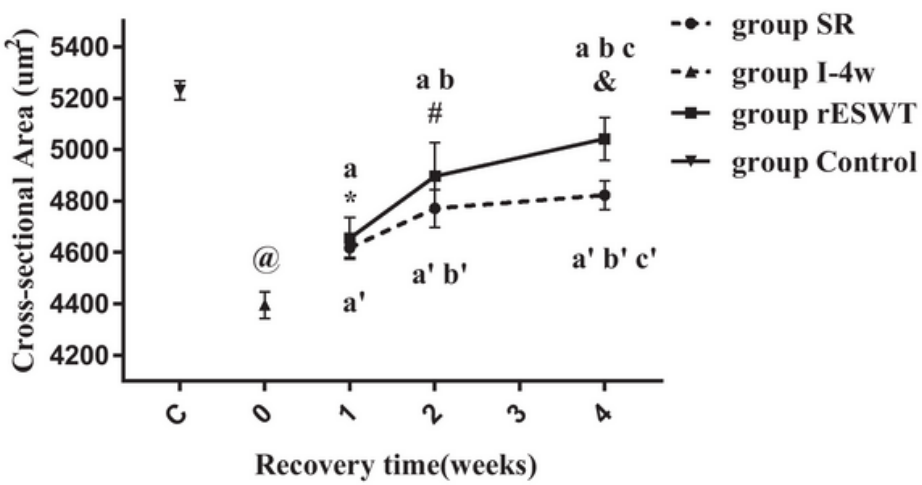

Figure 4

(A) Morphological changes of the rectus femoris. (a)The cross-sectional area in group Control; (b)the cross-sectional area in group I-4w; (c-e) the cross-sectional area after 1, 2, and 4 weeks of rESWT, respectively; $(\mathrm{f}-\mathrm{h})$ the cross-sectional area after one, two, and four weeks of self-recovery, respectively. Scale bar $=100 \mu \mathrm{m}$. (B) Effects of self-recovery and rESWT on the cross-sectional area of the rectus femoris. @P < .05 vs. group Control, aP < .05 vs. group l-4w, bP <.05 vs. group rESWT-1w, cP $<.05$ vs. group rESWT-2w, a'P $<.05$ vs. group l-4w, b'P $<0.05$ vs. group $S R-1 w, c^{\prime} P<0.05$ vs. group $S R-2 w ;{ }^{*} P<.05$ vs. group SR-1w, \#P <.05 vs. group SR-2w, \&P <.05 vs. group SR-4w. 
A
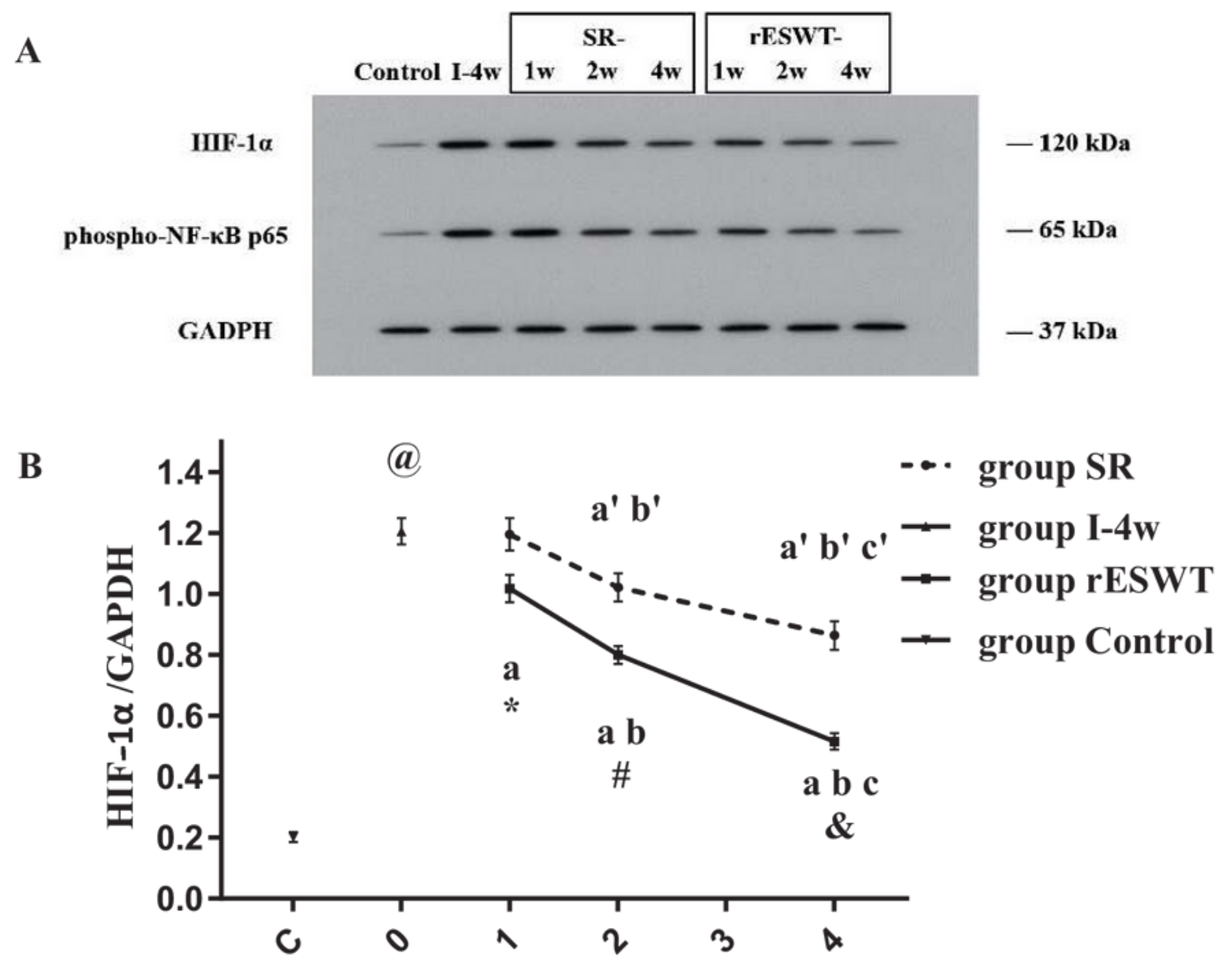

Recovery time(weeks)

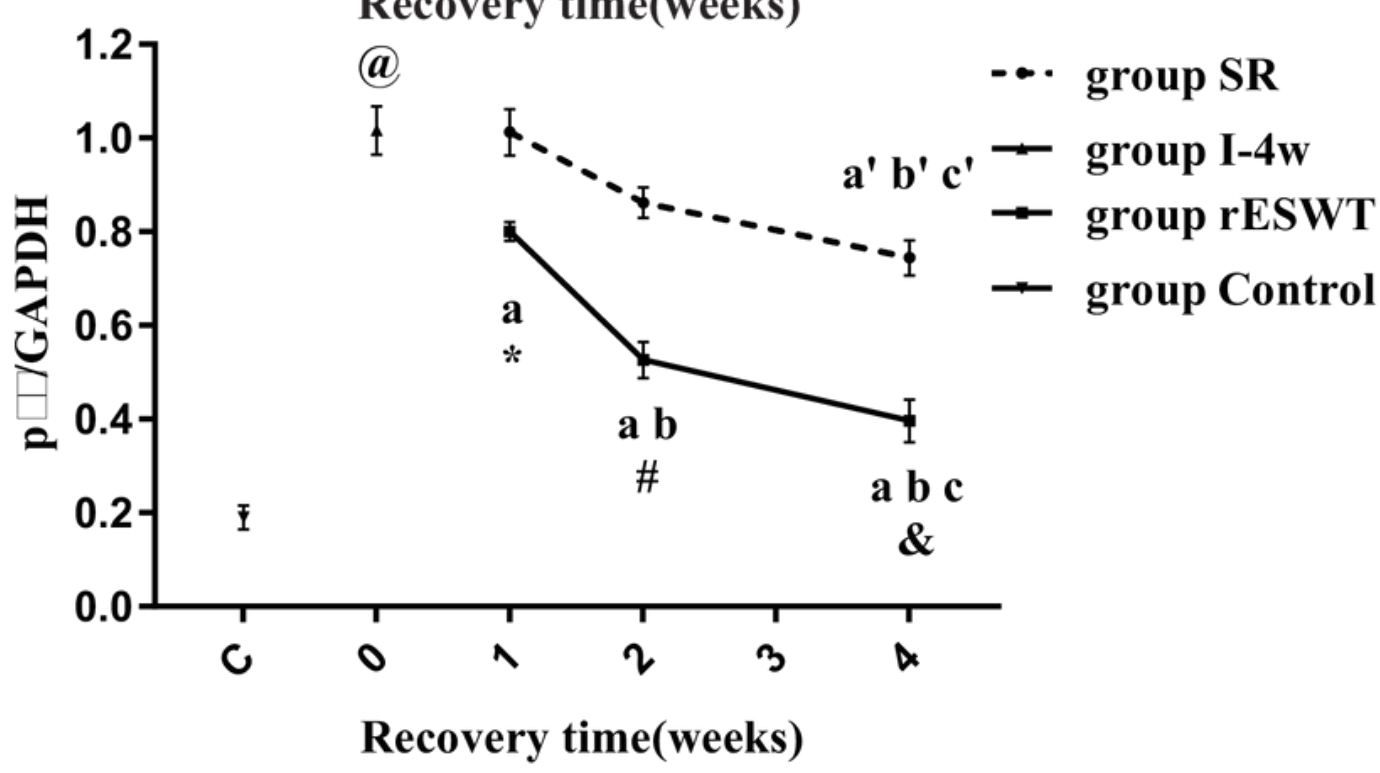

Figure 5

(A) The HIF-a/GAPDH and phospho-NF-kB p65/GAPDH of the rectus femoris in each group. (B) Effects of self-recovery and rESWT on the average protein levels for HIF-a and phospho-NF-KB p65. @P <.05 vs. group Control, aP < .05 vs. group l-4w, bP <.05 vs. group rESWT-1 w, cP <.05 vs. group rESWT-2w, a'P $<.05$ vs. group l-4w, b'P $<0.05$ vs. group SR-1 w, c'P $<0.05$ vs. group SR-2w; ${ }^{*} P<.05$ vs. group $S R-1 w$, \#P $<.05$ vs. group SR-2w, \&P < .05 vs. group SR-4w. 


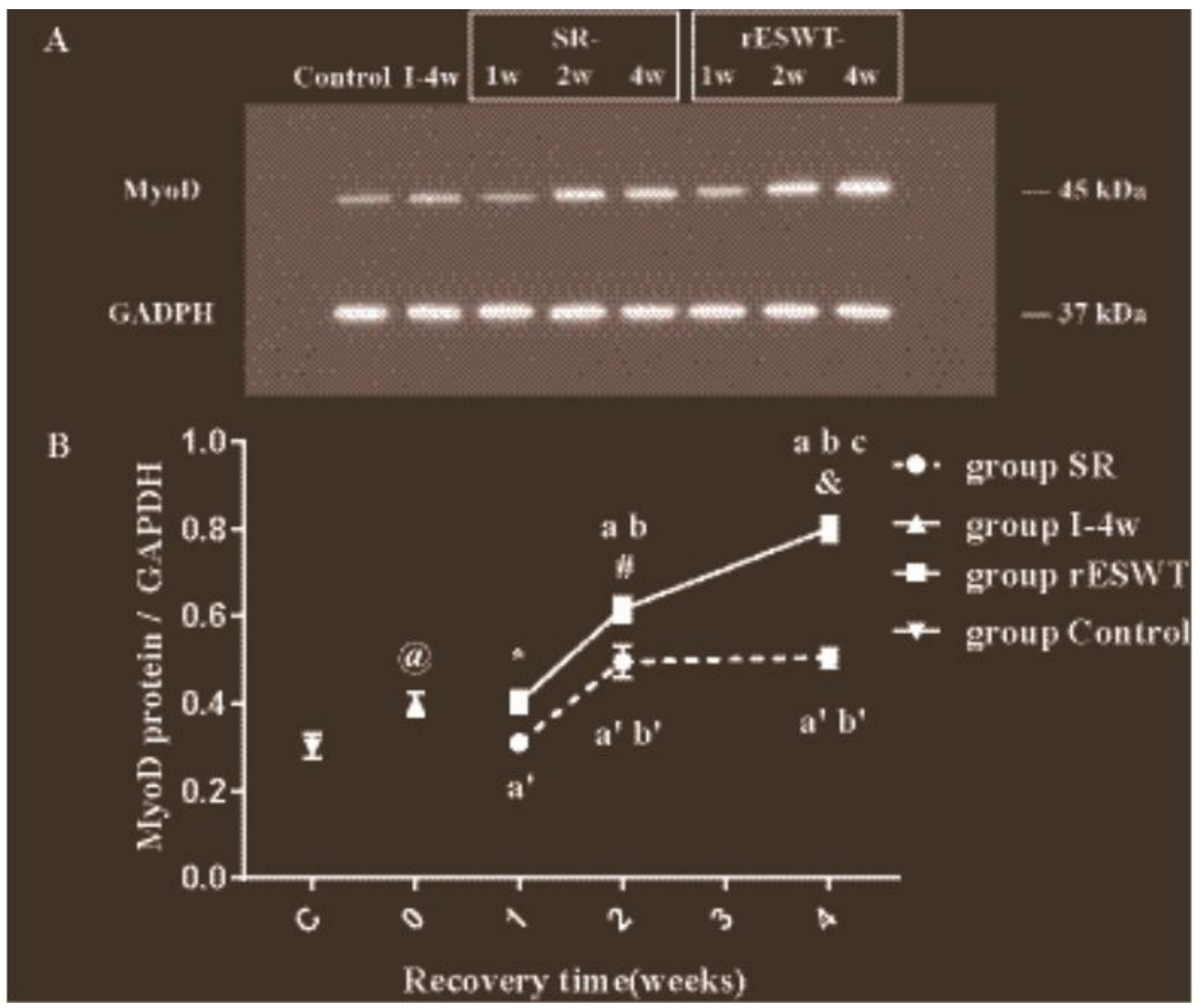

\section{Figure 6}

(A) The MyoD protein/GAPDH of the rectus femoris in each group. (B) Effects of self-recovery and rESWT on the average protein levels for MyoD. @P $<.05$ vs. group Control, aP $<.05$ vs. group I-4w, bP $<.05$ vs. group rESWT-1w, cP $<.05$ vs. group rESWT-2w, a'P $<.05$ vs. group $I-4 w, b^{\prime} P<0.05$ vs. group $S R-1 w, c^{\prime} P<$ 0.05 vs. group SR-2w; ${ }^{*}<<.05$ vs. group SR-1w, \#P<.05 vs. group SR-2w, \&P<.05 vs. group SR-4w. 